\title{
Effect of Modulation Index of Mach-Zehnder Modulator on Single Sideband Radio over Fiber System
}

\author{
Chao Jiang, Xiaogang Chen*, Zhaoxu Liu \\ College of Science \\ China Three Gorges University (CTGU) \\ Yichang, China \\ e-mail: cxg826@yahoo.com.cn (corresponding author: Xiaogang Chen)
}

\begin{abstract}
The effect of the modulation index of Mach-Zehnder modulator on single sideband (SSB) radio over fiber (RoF) system is investigated, and two different data modulation schemes are considered. The quantitative results suggest that there exist an optimum modulation index, and the system performance could be improved if the data signal is modulated on only optical carrier or sideband.
\end{abstract}

Keywords-Microwave photonics; Radio over fiber; Single sideband modulation; Modulation index;

\section{INTRODUCTION}

The demand of the wideband radio access services makes the use of the millimeter band radio necessary for the future wireless telecommunication ${ }^{[10,11]}$. The $40-70 \mathrm{GHz}$ millimeter (mm)-wave becomes the first choice and is preferred to be transmitted by the optical fiber than by air or metal wave guides because of the high loss in air and the expensive value of the metal guide before it is distributed to the user by the antenna via the air. To transmit the mmwave signal in the fiber, it must be modulated onto the light wave first, which is called the optical mm-wave signal. So, the optical modulation of the mm-wave signal onto light wave is an essential technique in the radio-over-fiber (RoF) system. The RoF technology can be combined with the advantages of fiber optic transmission and millimeter-wave, alleviate the problem of limited transmission distance of wireless millimeter wave systems ${ }^{[1-4]}$. The millimeter-wave signal is one of the key technologies for achieving the millimeter-wave the RoF system of generating high-quality. At present has the way of millimeter wave signal to be many and varied ${ }^{[6,7]}$, modulates through the exterior modulator obtains single sideband (SSB) and optical carrier suppression (OCS) light carries a millimeter signal the procedure to come into favor ${ }^{[12,13]}$.

In this paper, we perform simulation investigation of two SSB RoF systems with different data modulation scheme, and the effect of modulation index (MI) of MachZehnder modulator (MZM) on the system performance is quantitative analyzed and discussed. The obtained results can provide assistance for RoF system design.

\section{PRINCIPLE OF SSB ROF SYSTEM}

In ROF system, the output amplitude of DSB modulation is periodic fluctuation with the fiber length. Now, theoretical analysis and experimental results have shown that SSB modulation could eliminate this effect. The principal diagram of SSB optical millimeter-wave generation and transmission is shown in Figure 1.

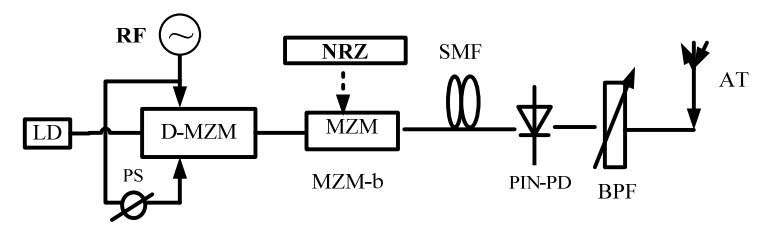

Figure 1. Schematic diagram of traditional SSB RoF system. LD: Laser diode, MZM: Mach-Zehnder modulator, RF: Radio frequency, PS: Phase shifter, SMF: Single mode fiber, PIN-PD: PIN Photoelectric detector, EDFA: Erbium doped fiber amplifier, BPF: Band pass filter, AT: Antenna terminal.

The output of the laser can be expressed as $E(t)=E_{0} \exp \left(\mathrm{j} \omega_{\mathrm{c}} \mathrm{t}\right)$, Where $E_{0}$ and $\omega_{\mathrm{c}}$ is optical carrier amplitude and angular frequency, respectively. The output electric field of the D-MZM modulator is:

$$
E_{\text {out }}(t)=\frac{E_{i}}{2}\left\{\exp \left[j \frac{\pi V_{1}(t)}{V_{\pi}}\right]+\exp \left[j \frac{\pi V_{2}(t)}{V_{\pi}}\right]\right\} \exp \left(j \omega_{c} t\right)
$$

Where $\quad V_{i}(t)=V_{d c i}+V_{R F} \cos \left(\omega_{R F} t+\phi_{i}\right), i=1,2$ represents drive signal applied to each arm of the device and $V_{\pi}$ is the switching voltage.

Various implementations of SSB optical subcarrier modulation (SCM) have been recently demonstrated. Among them, the SSB SCM generation based on a DDMZM offers one of the simplest methods to allow dispersion-tolerant transmission.

As shown in Figure 1. The implementation of SSB modulation is to use a D-MZM biased at quadrature point $(\gamma \pi=\pi / 2)$, in which a RF signal is split into two branches, which drive the two arms of the MZM with $\varphi_{2}-\varphi_{1}=\pi / 2$, and $V_{d c 1}-V_{d c 2}=V_{\pi} / 2$. Then we can implement the SSB modulation.

After using up Optical filter (OF) filters out the higher harmonic, can obtain the SSB signal ${ }^{[3]}$, the expression of SSB signal is: 


$$
\begin{array}{r}
E_{S S B}(0, t)=\frac{E_{0}}{\sqrt{2}} \cos \left(\frac{\pi}{4}\right) J_{0}(m) \exp \left[j\left(\omega_{c} t+\phi_{d}\right)\right]+ \\
\frac{E_{0}}{\sqrt{2}} J_{1}(m) \exp \left[j\left(\omega_{c} t+\omega_{R F} t+\phi_{d}+\phi_{p}+\frac{\pi}{2}\right)\right]
\end{array}
$$

Where $m=\pi V_{R F} / V_{\pi}$ is the modulation index of MZM modulator, and $\mathrm{J}_{\mathrm{n}}(\mathrm{m})$ is the Bessel function of first kind and order $n$. In equation (2), the first term is the optical carrier component, and the second term is optical millimeter wave sideband component. We can find that the frequency interval of the two components is $\mathrm{f}_{\mathrm{RF}}$.

Furthermore, as optical carrier and optical millimeter wave sideband are detected by an ideal photo-detector (PD), the temporal expression of the detected current can be calculated from the envelope of the incident optical signal and expressed as:

$$
I_{S S B}(t)=\frac{E_{0}^{2} J_{0}(m) J_{1}(m)}{2 \sqrt{2}} \cos \left(\omega_{R F} t+\phi_{p}+\frac{\pi}{2}\right)
$$

In order to improve the system performance of SSB RoF link, the modified approach is employed. The key issue of the modified scheme is that data signal is modulated on only optical carrier of sideband after separation of carrier and sideband. The principal diagram of the improved SSB RoF system is shown in Figure 2.

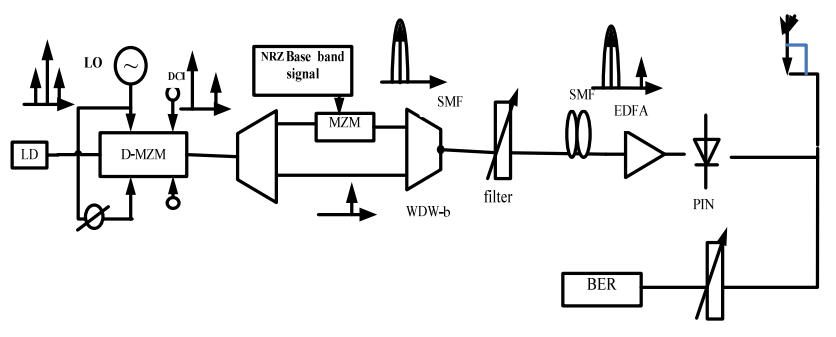

Figure 2. Schematic diagram of improved SSB RoF system

\section{SIMULATION AND DISCUSSION}

To study the influence of the modulation index of MZM on system performance in the SSB ROF link, simulation calculation has been respectively performed for the following two different situations by using the commercial software package OptiSystem.

In the first situation, the principal diagram is shown as Figure 1. Non-return-to-zero (NRZ) data signal is modulated on two optical carrier and sideband simultaneously, and incoherent detection is employed to achieve simple and costeffective receiver. The system parameters are set as follows: The center frequency of LD is $193.1 \mathrm{THz}, 3 \mathrm{~dB}$ bandwidth of $\mathrm{LD}$ is $10 \mathrm{MHz}$. The data rate is $2.5 \mathrm{Gbit} / \mathrm{s}$ and the RF frequency is $40 \mathrm{GHz}$. We assume modulator is the ideal LiNb Mach-Zehnder Modulator and the switching voltage of MZM is $4 \mathrm{~V}$. The attenuation of SMF can be compensation by EDFA. The fiber dispersion is set to $16.75 \mathrm{ps} / \mathrm{nm} / \mathrm{km}$. The responsivity of photo-detector is $1 \mathrm{~A} / \mathrm{W}$. The output signal of $\mathrm{PD}$ will be filtered by the band-pass filter (BPF) with center wavelength of $40 \mathrm{GHz}$.

For a distribution system with a transmission distance of $30 \mathrm{Km}$ over a SMF link, the maximum $\mathrm{Q}$ factor versus modulation voltage of MZM in the traditional SSB RoF link is plotted in Figure 3. It is clearly seen that maximum $Q$ factor varies with the tuning of modulation voltage of MZM, and there exist an optimum modulation voltage (1.4V) corresponding to best system performance $(\mathrm{Q}$ factor $=8.1)$.

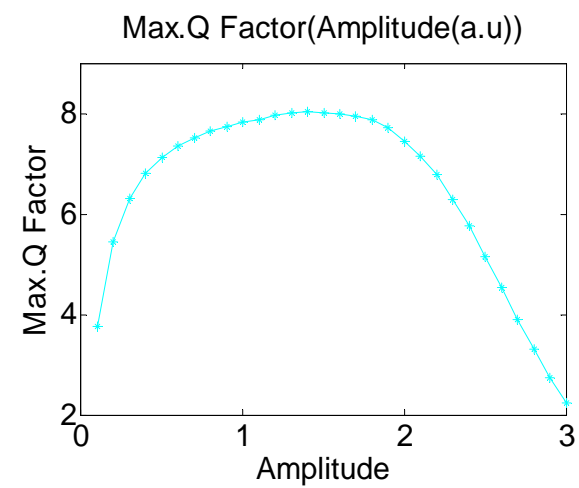

Figure 3. Maximum Q Factor versus modulation voltage in the traditional SSB RoF system

For the traditional SSB RoF link with optimum modulation voltage $1.4 \mathrm{~V}$, the maximum $\mathrm{Q}$ factor versus transmission distance is plotted in Figure 4. As can be seen from the figure, to achieve $\mathrm{Q}$ factor $=6\left(\mathrm{BER}=10^{-9}\right)$, the maximum transmission distance is about $38 \mathrm{Km}$.

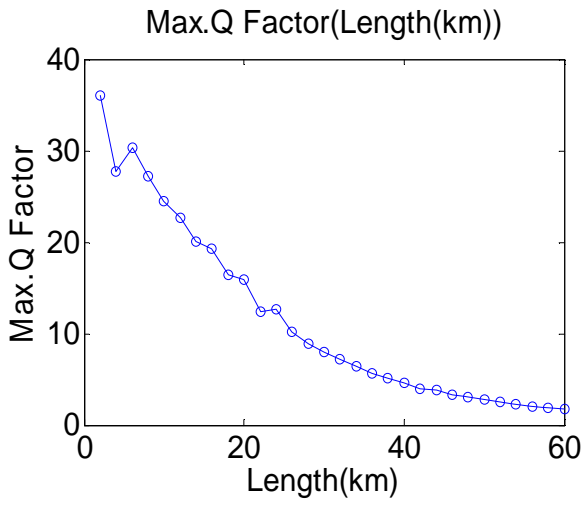

Figure 4. Maximum Q Factor versus transmission distance in the traditional SSB RoF system with optimum modulation index.

In the second situation, the principal diagram is shown as Figure 2. NRZ data signal is modulated on one of two optical millimeter wave sidebands, and the system parameters are set as those of the first situation, incoherent detection is also employed.

For a distribution system with a transmission distance of $30 \mathrm{Km}$ over a SMF link, the maximum $\mathrm{Q}$ factor versus modulation voltage of MZM in the improved SSB RoF link is plotted in Figure 3. As can be also from the figure, there exist an optimum modulation voltage corresponding to best 
system performance, and the optimum modulation voltage and maximum $\mathrm{Q}$ factor is 1.3 and 37.5, respectively.

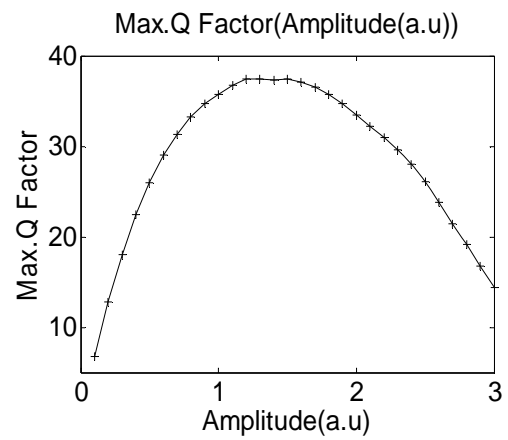

Figure 5. Maximum $\mathrm{Q}$ Factor versus modulation voltage in the improved SSB RoF system

For the improved OCS RoF link with optimum modulation voltage $1.3 \mathrm{~V}$, the maximum $\mathrm{Q}$ factor versus transmission distance is plotted in Figure 4. It is clearly seen that the maximum transmission distance is about $120 \mathrm{Km}$ to achieve $\mathrm{Q}$ factor $=6\left(\mathrm{BER}=10^{-9}\right)$.

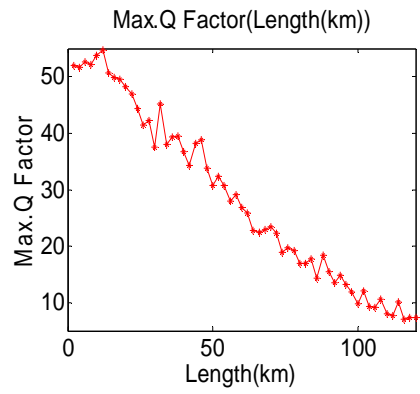

Figure 6. Maximum Q Factor versus transmission distance in the improved SSB RoF system with optimum modulation index

The eye diagram of improved SSB RoF system with different transmission distance is shown in Figure 7, and the transmission distance is $0 \mathrm{~km}, 20 \mathrm{~km}, 40 \mathrm{~km}, 60 \mathrm{~km}, 90 \mathrm{~km}$, $120 \mathrm{~km}$, respectively. It can be seen from the figure that the eye diagram was obvious and opened even transmission distance reach astonishing $120 \mathrm{~km}$. however, the eye diagram was not so obvious as the transmission distance reach $140 \mathrm{~km}$

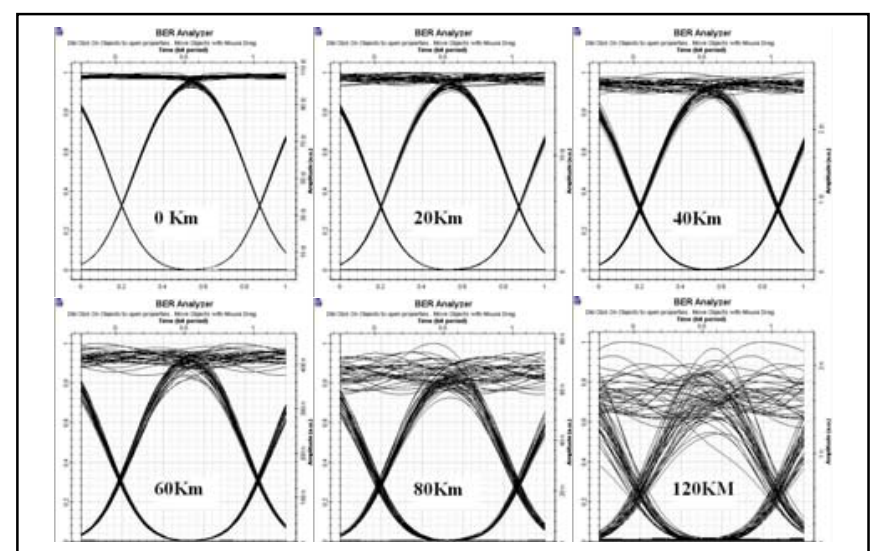

Figure 7. The eye diagram of improved OCS RoF system with different transmission distance.

\section{CONCLUSIONS}

In conclusion, the influence of modulation index of MZM on system performance of SSB RoF link using different data signal modulation scheme is quantitative analyzed and simulated. The theoretical and simulation results show that system performance could be enhanced by tuning modulation voltage of MZM, and the power of optical carrier is equal to that of sideband. In addition, transmission distance could be extended markedly if the data signal is modulated on only optical carrier or sideband.

\section{REFERENCES}

[1] Jiang Tao , Huang De xiu , Zhang Xin liang et al . . A full-duplex 60 GHz-band radio over fiber system [J] . Acta Optica Sinica ,2008 , 28 (1) : $37 \sim 42$

[2] Hu Li liang, Chen Lin , Yu Jianjun et al . . A novel scheme for optical millimeter wave generation based on double-Sideband modulation [J] . Acta Optica Sinica , 2008 , 28 (2) : 239 242

[3] Fang Zu jie , Ye Qing , Liu Feng et al . . Progress of millimeter wave subcarrier optical fiber communication technologies $[\mathrm{J}]$.Chinese $\mathrm{J}$. Lasers , 2006, 33 (4) : 481 488.

[4] J . X. Ma , J . Yu , C. G. Yu et al . . Fiber dispersion influence on tran smission of the optical millimeter waves generated using LN-MZM intensity modulation [J]. IEEE J.Light wave Technol . ,2007 , 25 (11) : 3244 3255

[5] J.J.Yu,Z.S.Jia,L.L.Yi et al..Optical millimeter-wave generation or upconversion using external modulators[J].I E E E Photon. Technol.Lett. .2006,18(1):265 267.

[6] C. Lim , C. Lin , M. Attygalle et al . . Analysis of optical carrier-tosideb and ratio for improving transmission performance in fiber-radio links [J] .IEEE Trans . Microw . Theory Tech . , 2006 ,54 (5) : 2181 2187

[7] Tao Wang, Qingjiang Chang, Yikai Su. Generation of linearzed optical single side band signal for broadband radio over fiber systems[J ] . CHIN. OPT . LETT . , 2009 , 7 (4) : 339 343

[8] Jianjun $\mathrm{Yu}$, Mingfang Huang, Zhensheng Jia et al . . A novel scheme to generate single-sideband millimeter-wave signals by using low- 
frequency local oscillator signal [J] .IEEE Photon.Technol . Lett . , 2008,20 (7) : 478 480

[9] P. C. Peng, C. T. Lin , W. J . Jiang et al . Improvement of transmission in fibre wireless system using semiconductor laser amplifier [J] . Elect ron. Lett . , 2008 , 44 (4) : 520 521

[10] Cox III. CH, Ackerman EI, Betts GE, Prince JL. Limits on the performance of RF-over-fiber links and their impact in the device design. IEEE Trans Microwave Theory Tech 2006;54:906-20.
[11] Choi ST, Yang KS, Nishi S, Shimizu S, Tokuda K, Kim YH. A 60$\mathrm{GHz}$ point-to-point millimeter-wave fiber-radio communication system. IEEE Trans Microwave Theory Tech 2006;54:1953-60.

[12] Ma J, Yu J, Yu C, Sang X, Xin X. Transmission performance of the optical mm-wave generated by double sideband intensity-modulation. Opt Commun 2007;280:317-26.

[13] Yu J, Jia Z, Yi L, Su Y, Zhang G-K, Wang T. Optical millimeterwave generation or up-conversion using external modulators. IEEE Photon Thnol Lett 2006;18:265-7. 